\title{
O AUTORRELATO NA PESQUISA EM PSICOLOGIA DA SAÚDE: Desafios metodológicos
}

\author{
Self-report in health psychology research: M ethodological challenges
}

\author{
Marina Kohlsdorfa, Áderson Luiz da Costa Junior ${ }^{b}$ \\ a Mestre em Processos de D esenvolvimento Humano e Saúde pela Universidade de Brasília, Brasília, DF - Brasil, e-mail: \\ marinak@unb.br \\ ${ }^{\text {b }}$ D outor em Psicologia pela Universidade de Brasília, Pós-D outorado pela Universidade Estadual de Campinas, Professor \\ adjunto no Instituto de Psicologia da Universidade de Brasília, Brasília, D F - Brasil, e-mail: aderson@ unb.br
}

\section{Resumo}

D iversas áreas de conhecimento, como a Psicologia, empregam o autorrelato em pesquisas, sob a forma de entrevistas, questionários, inventários e escalas. Neste trabalho, são apresentadas reflexões sobre os desafios implicados no uso de autorrelato, em especial no campo de atuação da psicologia da saúde que focaliza processos de enfrentamento e adesão ao tratamento. 0 comportamento verbal é reforçado pela comunidade social em que o indivíduo se encontra e o autorrelato somente pode ocorrer a partir da auto-observação, pois se encontra associado a estímulos e comportamentos aos quais somente 0 falante tem acesso. 0 autorrelato não garante que haja correspondência exata entre o comportamento real e aquele verbalizado, por algumas razões: (a) o comportamento relatado difere do comportamento real porque eventos encobertos não são reconhecidos de forma legítima; (b) o comportamento relatado não é o real, mas o comportamento socialmente desejável, considerando influências da história de reforçamento do indivíduo; e (c) o participante não compreende inteiramente os itens que compõem o instrumento utilizado, resultando em significações distintas para participante e pesquisador. A fim de minimizar a discrepância entre o dado obtido a partir do autorrelato e 0 comportamento real, é importante que o pesquisador em psicologia da saúde invista no treinamento e preparo para a coleta de dados e utilize metodologias combinadas (tais como observação do comportamento e aplicação de mais de um tipo de instrumento, entre escalas, inventários e questionários), realizando medidas complementares sobre o comportamento ou processo estudado.

Palavras-chave: Autorrelato. Psicologia da saúde. Enfrentamento. Adesão. 


\begin{abstract}
Several fields, such as Psychology, use self-report in researching, as interviews, questionnaires, inventories and scales. This paper presents some discussion about challenges concerning self-report, specially in Health Psychology and focusing coping and treatment adherence. Verbal behavior is reinforced by social community and self-report can only occur because os self-observation, since it's associated to stimulus and behaviors to which only the speaker has access. Self-report does not assure a full correspondence between actual behavior and verbalized behavior, according to some reasons: (a) the reported behavior differs from actual behavior because private events are not legitimally recognized; (b) reported behavior is not equal to actual behavior, but it is the socially desirable behavior, considering influences from individual's reinforcement history; and (c) the participant does not fully understand the items that comprise the instrument, consequently there are different meanings for the participant and the researcher. In order to decrease discrepancy between data from self-report and actual behavior, the researcher in Health Psychology should invest in training concerning data collection and should also use combined methodologies (such as behavior observation and more than one single type of instrument, between scales, inventories, and questionnaires), using complementary measures related to the focused behavior or process.
\end{abstract}

Keywords: Self-report. Health Psychology. Coping. Treatment adherence.

\section{INTRODUÇÃO}

Em Análise do Comportamento, "relatar" constitui um comportamento verbal, incluído na categoria de operante verbal denominada tato e, portanto, emitido sob controle de um contexto constituído por um conjunto de estímulos discriminativos integrados (D e Rose, 2001). A propriedade característica do tato é o controle singular que elementos do meio exercem sobre a forma da resposta, ou seja, o relato é diretamente controlado pelas contingências ambientais. Sendo comportamento verbal, ocorre a partir do reforçamento disponibilizado pela comunidade verbal em que o indivíduo se encontra inserido e se torna eficaz somente a partir da mediação de outras pessoas: um episódio verbal total é constituído pelo comportamento do falante e do ouvinte juntos.

A o considerar o relato verbal como tato, D e Rose (2001) destaca que o grau de controle de um estímulo sobre o relato depende do grau de acesso do sujeito ao estímulo discriminativo, do grau de acesso da comunidade verbal aos estímulos durante o estabelecimento do repertório e dos procedimentos utilizados pela comunidade para manter tal repertório. D estaca-se a importância da comunidade verbal não apenas para a aquisição, por parte do indivíduo, do comportamento verbal em si, mas principalmente para definir quais comportamentos serão mantidos por serem socialmente desejáveis (em outras palavras, por reforçarem comportamentos do ouvinte) e quais serão punidos, tendo sua probabilidade de ocorrência reduzida.

De acordo com Skinner (1957/ 1978), o comportamento verbal é determinado por vários elementos ao mesmo tempo, entre estímulos, reforçamentos e punições. D esta forma, ao considerar 0 autorrelato como o comportamento verbal acerca do comportamento do próprio indivíduo, as amplas contingências associadas ao autorrelato (estímulos, reforçamentos, punições) devem ser consideradas, implicando alguns desafios que se impõem no trabalho epesquisa que focalizam esse comportamento específico.

Muitas áreas de conhecimento utilizam 0 autorrelato sob aformadeentrevistas, questionários, inventários e escalas, para obtenção de dados de pesquisa. $\mathrm{Na}$ Psicologia, diversas subáreas e abordagens utilizam tais instrumentos para estudos, análises experimentais e clínicas. Neste trabalho, elaboram-se reflexões sobre os desafios implicados no uso do autorrelato enquanto instrumento de pesquisa, com ênfase em sua utilização como parte de metodologias de pesquisa em psicologia da saúde - área definida por Matarazzo (1980) como o 
conjunto de contribuições educacionais, científicas e profissionais específicas da Psicologia para promover e manter a saúde, prevenir e tratar a doença, a partir da identificação de fatores etiológicos e diagnósticos da saúde.

\section{0 autorrelato como instrumento para conhecer eventos privados}

Skinner (1957/ 1978) destaca que o comportamento acerca do comportamento acarreta a mesma dificuldade que 0 conhecimento acerca do conhecimento. Especificamente, 0 autorrelato somente pode ocorrer a partir da auto-observação, porque está ligado a estímulos e comportamentos com os quais somente 0 falante pode ter certo tipo de relação, ou seja, somente ele tem acesso a seus eventos privados, comportamentos encobertos e comportamentos abertos não observáveis por outras pessoas.

No caso do atendimento clínico e da pesquisa a partir do autorrelato, são apresentados de imediato alguns problemas: (a) o pesquisador/ terapeuta não pode apontar a quais estímulos deve recorrer para a previsão e controle do comportamento; (b) sem acesso a estímulos privados, uma comunidade verbal reforça determinados comportamentosverbais enão reforça outros, muitas vezes criando contingências em que 0 autorrelato fidedigno ao comportamento não é reforçado ou, ainda, é punido. Um exemplo dessa situação é a nomeação equivocada de eventos privados (raiva, tristeza, ansiedade) por crianças aprendendo a falar; outro exemplo clássico desse contexto ocorre quando 0 indivíduo deliberadamente relata um comportamento diferente do ocorrido (ou, em linguagem popular, mente). A partir do contexto supracitado, ficam evidentes as dificuldades inerentes ao autorrelato, sejaporfaltadereconhecimento do comportamento privado, seja porum relato deliberadamente diferente do comportamento efetivamente executado.

Além disso, o conhecimento sobreo próprio comportamento depende da auto-observação e da exposição do indivíduo a estímulos decorridos de seu próprio comportamento. Consequentemente, conforme De Rose (2001), esse autoconhecimento pode ser problemático em decorrência do fato de que 0 indivíduo pode ignorar muitos aspectos a respeito de si mesmo, comprometendo a precisão de seus relatos verbais e as inferências que o pesquisador pode fazer a respeito desses dados. É possível que 0 indivíduo ignore que fez/ faz algo específico, a partir da ausência de respostas de auto-observação; pode ainda atribuir diferentes topografias a comportamentos auto-observados (que podem não corresponder totalmente à realidade objetiva do comportamento); pode, também, não saber que está fazendo alguma coisa ou não reconhecer as variáveis que controlam seu comportamento, como muitas vezes evidenciado na prática psicoterápica e clínica.

Ainda em relação à auto-observação, cabe ressaltar que a sociedade (enquanto comunidade verbal) estabelece contingências que levam 0 indivíduo a se auto-observar e relatar seu próprio comportamento, ou seja, o controle de estímulo somente pode ser estabelecido por meio do reforço social. D eacordo com D eRose (2001), pode ocorrer distorção do autorrelato a partir do momento em que 0 tato é mantido por reforço generalizado e respostas verbais com conteúdos específicos tendem a receber maior quantidade desse reforço generalizado, produzindo relatos de acordo com 0 que é socialmente desejável e nem sempre com 0 modo como o indivíduo realmente se comporta.

\section{0 autorrelato e a pesquisa em psicologia da saúde}

De Rose (2001) e Skinner (1957/ 1978) destacam que o relato é uma das fontes de dados mais amplamente utilizadas não apenas na Psicologia, mas em ilimitadasáreas de conhecimento em Ciências Sociais. A fidedignidade dainformação, segundo os autores supracitados, éum ponto crucial, bem como a natureza dos dados obtidos. Desta forma, o exame de atitudes, relatos e opiniões, a partir de questionários, escalas e inventários, é de grande valia ao informar a percepção do indivíduo sobre diversos aspectos de seu comportamento e do comportamento alheio, mas háum problema crucial no status do "relato verbal": até que ponto 0 comportamento relatado corresponde ao comportamento efetivo? 0 dado obtido seria a tendência do comportamento, a percepção do falante sobre seu próprio comportamento ou, ainda, o comportamento desejável socialmente?

Em Psicologia da Saúde, os pesquisadores também se interessam pelo relato do comportamento, evento, estado e fenômeno que 
não podem ser acessados de forma fácil ou direta. 0 autorrelato se constitui, assim, a base de entrevistas clínicas, escalas, inventários, diários, levantamentos, questionários, avaliações padronizadas, entre outros. $\mathrm{Na}$ Análise do Comportamento, em muitos casos, a observação direta de comportamentos somente é possível a princípio, mas impossível na prática, restando ao pesquisador recorrer a relatos verbais sobre o comportamento do próprio indivíduo (De Rose, 2001). Esse contexto é compartilhado pelos estudos em Psicologia da Saúde, em que o indivíduo é solicitado a responder entrevistas ou avaliações padronizadas, relatando seu próprio comportamento em situações específicas de cuidados de promoção da saúde e prevenção de doenças, contextos em que, em muitos casos, não é possível estabelecer uma observação diretado comportamento poroutras pessoas que não o próprio indivíduo.

Mesmo em situações que não sejam percebidas como ameaçadoras ou punitivas, existe sempre a possibilidade de que alguns indivíduos não revelem seus comportamentos, em decorrência de uma história de reforçamento (à qual todos são submetidos, a partir da vivência em sociedade) que prioriza relatos socialmente desejáveis. Além disso, 0 autorrelato está sujeito a limitações de atenção, memória, acessibilidade e mesmo efeitos colaterais e iatrogênicos da medicação. A menos que o objeto de estudo seja formado também pelas percepções do indivíduo em seu processo saúde-doença, seria preferível observar diretamente 0 comportamento de interesse e analisar contingências associadas a tal comportamento, mas isto geralmente não é possível.

Além disso, De Rose (2001) salienta que, enquanto comunidade verbal, somos capazes de descrever contingências de reforçamento, mas não há evidência de que descrevemos verbalmente todas as contingências que afetam o comportamento. De modo semelhante, na pesquisa em Psicologia da Saúde, diversos instrumentos utilizados focalizam o relato do comportamento, mas não descrevem todas as contingências associadas ao comportamento em foco, nem exploram quais contingências mantêm o comportamento (mesmo porque os objetivos de inventários e pesquisas poderiam ser modificados, ao explorar contingências que mantêm 0 comportamento em questão).

Ao utilizar o autorrelato para coleta de dados, o pesquisadorem PsicologiadaSaúdeencontrase numa situação em que o falante (participante da pesquisa) esteve e/ ou está em contato com um contexto inacessível ao ouvinte (pesquisador). Quando uma observação direta de comportamentos em ambiente de cuidados com a saúde não é possível, a forma de entrar em contato com a realidade vivenciada pelo falante e as contingências associadas ao comportamento em questão é a partir do autorrelato do indivíduo, expresso em inventários, entrevistas, questionários, diários e escalas.

Modificarcontingênciasno processo saúdedoença somente é possível a partir da compreensão sobre como o indivíduo percebe e vivencia seu contexto imediato, fato que se torna possível a partir do autorrelato. Para os propósitos de reflexão acerca do uso do autorrelato em pesquisas na área de Psicologia da Saúde, foram escolhidos dois processos bastante estudados na área, nas últimas décadas, para ilustrar algumas dificuldades metodológicas no uso do autorrelato: a adesão ao tratamento e 0 enfrentamento de contextos estressores.

\section{Adesão ao tratamento}

0 termo adesão ao tratamento tem sido utilizado para designar o grau de coincidência entre os comportamentos do indivíduo eas recomendações terapêuticas do profissional de saúde (Epstein \& Cluss, 1982). Geralmente, o que se percebe é um conjunto decomportamentoscorrespondentesamais deumarecomendação, possibilitando avaliaraadesão segundo diferentes níveis. Arruda (2002), por exemplo, relata que as medidas de adesão se relacionam ao uso demedicamentos, comparecimento a consultas, controle do estado físico, seguimento de dietaalimentare recomendações médicas, suspensão, modificação ou inclusão de atividades físicas e alterações de rotina, considerando que as exigências de cada diagnóstico estabelecem quais procedimentos serão adotados.

A Psicologia da Saúde tem estudado sistematicamente a magnitude do problema de uma adesão insatisfatória ao tratamento há, pelo menos, 20 anos, considerando a influência de fatores como complexidade do tratamento, frequência de cuidados diários, restrições alimentares e/ ou físicas, riscos e efeitos colaterais associados à medicação, eficácia do tratamento, crenças e aspectos culturais do indivíduo e de seu grupo social (Malerbi, 2000). Nesse contexto, o ambiente social do indivíduo influencia diretamente níveis de adesão, ao fornecer reforços contingentes aos comportamentos correspondentes a recomendações de cuidado. 
Arruda (2002) descreve que, de modo geral, são utilizadas categorias não-formais para conceituar e medir a adesão, que é caracterizada em grande parte por meio do autorrelato do indivíduo em diferentes momentos do tratamento.

\section{Enfrentamento}

Por enfrentamento (tradução do original, em inglês, coping), entende-se o conjunto de processos utilizados para que um indivíduo se adapte a uma situação estressora, incluindo respostas frente a uma crise para administrar, reduzir ou tolerar as demandas criadas por tal situação (Lazarus \& Folkman, 1984). Ainda considerando Folkman, Lazarus, Gruen e DeLonguis (1986), o enfrentamento compreende o conjunto de esforços para lidar com algo que é percebido pelo indivíduo como ameaça iminente, percebido como sobrecarga. Segundo Antoniazzi, Dell'Aglio e Bandeira (1998), durante a vida os indivíduos precisam lidar com acontecimentos que exigem respostas adaptativas, sugerindo a noção de estilos de enfrentamento (comportamentos comumente utilizados, de modo geral, em situações aversivas) e de estratégias de enfrentamento (comportamentos específicos como respostas a estressores específicos).

Lazarus e Folkman (1984) apontam dois embasamentos principais para as estratégias de oping: focalização no problema (utilizadas quando a situação é percebida pelo indivíduo como passível de mudanças) e focalização na emoção (quando a situação aversiva é percebida como imutável). A literatura aponta um aumento nos estudos e pesquisas em Psicologia da Saúde a respeito das estratégias de enfrentamento adotadas em especial por pacientes portadores de doenças crônicas, embora ainda haja uma lacuna entre pesquisas básicas sobre enfrentamento e pesquisas sobre intervenções diretas em relação a estratégias de enfrentamento (Coyne \& Racioppo, 2000).

As estratégias de enfrentamento são comumente avaliadas a partir de instrumentos padronizados (Cerqueira, 2000; Seidl, Tróccoli \& Zannon, 2001) que utilizam o autorrelato do participante, mas existem problemas na fidedignidade das medidas de enfrentamento, a partir da variabilidade conceitual das respostas e mesmo da falta de clareza conceitual, ainda presente na literatura, sobre a definição e classificação de estratégias de enfrentamento.

\section{Dificuldades metodológicas na pesquisa com autorrelato em psicologia da saúde}

Para modificar contingências no processo saúde-doença, éfundamental compreendero contexto conforme vivenciado e percebido pelo indivíduo, ou seja, inventários, questionários, diários, escalas e entrevistas são instrumentos bastante úteis para apreender a realidade do indivíduo a partir de sua própria percepção, que inclusive amplia o que é observado externamente. Entretanto, alguns desafios metodológicos não podem ser ignorados.

D e forma geral, é possível destacar três principais dificuldades na utilização do autorrelato em pesquisa na área de Psicologia da Saúde:

(a) o comportamento relatado difere do comportamento real porque eventos encobertos não são reconhecidos de forma legítima;

(b) o comportamento relatado não é o real, mas o comportamento socialmente desejável, considerando influências da história de reforçamento do indivíduo;

(c) o participante não compreende inteiramente os itens que compõem 0 instrumento utilizado, resultando em significações distintas para participante e pesquisador. Cada um desses elementos é explorado em detalhes, a seguir.

1) 0 comportamento relatado não corresponde a comportamentos privados e encobertos.

D e acordo com D e Rose (2001), no caso de eventos privados, a comunidade verbal não tem acesso direto a quais estímulos são contingentes a respostas privadas; além disso, a própria imprecisão dos receptores sensoriais que respondem aos estímulos internos (proprioceptivos e interoceptivos) se constitui uma dificuldade adicional. Isso indica que as discriminações que 0 indivíduo desenvolve a respeito do mundo privado são, provavelmente, menos precisas que as discriminações a respeito do mundo externo. Por exemplo, indicadores somáticos leves de ansiedade 
(tais como alterações na frequência respiratória e suor) podem não ser efetivamente percebidos pelo indivíduo como mudanças significativas.

D esta forma, o autorrelato pode não ser correspondente ao comportamento real a partir de uma discriminação pobre a respeito do mundo encoberto ou privado do indivíduo, ou seja, eventos públicos e privados podem não ser perfeitamente correlacionados. No âmbito das pesquisas que investigam estratégias de enfrentamento, baseadas prioritariamente no autorrelato, esse contexto demanda cuidados por parte do pesquisador, tanto na escolha de metodologia quanto na análise dos dados obtidos, como descrito a seguir.

Cerqueira (2000) afirma que as pessoas, ao se confrontarem com situações aversivas, enfrentam-nas de formamais ou menos ansiogênica. As respostas de enfrentamento podem ter como objetivo neutralizar ou diminuir a situação aversiva, dominando a situação ou diminuindo a ativação fisiológica provocada pelo contexto, garantindo assim 0 equilíbrio e bem-estar psicológico do indivíduo. Entretanto, muitas vezes, a nomeação do comportamento pode ser equivocada por parte do participante da pesquisa. Skinner (1989/ 1991) exemplifica que o comportamento verbal "estou ansioso" pode não corresponder às manifestações fisiológicas que acompanham a ansiedade (mudança no tônus muscular, frequência cardiorrespiratória e pressão sanguínea), além de não possibilitar estabelecer quais contingências apresentam estímulos ansiogênicos.

Ainda conforme De Rose (2001), a privatividade de estímulos encobertos e privados e a observação privada de determinados comportamentos apresentam problemas: 0 investigador não pode, de imediato, apontar a quais estímulos deve recorrer para a previsão e controle do comportamento e não pode afirmar seguramente que 0 relato verbal do participante descreve fielmente os eventos privados associados a mudanças fisiológicas. 0 pesquisador não sabe, a rigor, quando e quanto pode confiar em um relato verbal. A melhoria das técnicas fisiológicas pode resolver eventualmente este problema, tornando públicos determinados eventos privados. Por exemplo, se fosse possível dizer precisamente que acontecimentos no interior do organismo controlam a resposta "estou triste", seria possível atingir o grau de previsão e controle característicos de respostas verbais a estímulos externos.
2) 0 comportamento relatado é 0 comportamento socialmente desejável, não o comportamento efetivo.

Skinner (1967) afirma que, sendo o comportamento verbal reforçado socialmente, 0 relato do indivíduo é classificado como "bom" ou "mau" ou ainda como "certo" ou "errado", sendo reforçado e punido de acordo. 0 comportamento do indivíduo é bom ou certo, geralmente, na medida em que reforça outros comportamentos do restante do grupo social; tal condição pode perpetuar 0 comportamento em questão não sendo mais reforçador ou aversivo. $\mathrm{O}$ bom comportamento geralmente é reforçado com elogios, gratificações, favores, enquanto o mau comportamento geralmente é punido com ameaças, repreensões, censuras.

Nos estudos e pesquisas em Psicologia da Saúde a respeito de enfrentamento, em que 0 autorrelato é a base da coleta de dados, a principal dificuldademetodológica residenaimpossibilidade de garantir relatos fidedignos de comportamento. Por exemplo, alguns indivíduos podem não relatar a adoção de estratégias de enfrentamento baseadas na emoção (como, por exemplo, demonstrar raiva para pessoas próximas, desejar vingança pela situação ou concentrar-se em eventos fantásticos ou impossíveis) devido a uma história de reforçamento que atribui um valor indesejável a tais comportamentos. Cerqueira (2000) destaca a importância de se avaliar o impacto de dados autorrelatados sobre a validade e fidedignidade das medidas, sugerindo a importância da obtenção de outros dados observados e de realizar pesquisas controladas em laboratório.

Considerando que uma das bases do autorrelato é a auto-observação, De Rose (2001) destaca que uma pessoa pode ignorar que fez/ faz algo específico, a partir da ausência de respostas de auto-observação; a situação do questionário ou da entrevista pode, eventualmente, fornecer pistas ou sondas para complementar o controle impreciso exercido pelo comportamento passado. Por outro lado, tais pistas ou sondas podem também atuar em conjunto com a história de reforço do indivíduo, induzindo sua resposta ao que é esperado pelo pesquisador. Muitas vezes, o pesquisador pode ainda inadvertidamente modelar o conteúdo dos relatos de tal modo que o sujeito venha a dizer o que o pesquisador espera ouvir.

Nos estudos sobre adesão a tratamento, verifica-se que o autorrelato é utilizado como uma 
das principais formas de acompanhar o processo de adesão. De acordo com Malerbi (2000), essa medida é sempre considerada suspeita, porque 0 comportamento pode estar sob controle das consequências (como a desaprovação do médico) e não da ocorrência do evento que deveria ser relatado (o comportamento de cuidado em saúde). A autora relata algumas estratégias que têm sido utilizadas em combinação com o autorrelato (como o uso de dispositivos dememória em glicosímetros) e destaca que, segundo a literatura, quando é solicitado o relato de comportamentos específicos, os dados são mais confiáveis.

Uma das dificuldades encontradas ao analisar comportamentos de adesão ao tratamento é relativa à falta de procedimentos eficazes para sua avaliação (Malerbi, 2000). De fato, indicadores biológicos podem ser eficientes medidas para avaliação da adesão, entretanto, os resultados fisiológicos nem sempre são adequados porque podem ser afetados por outros fatoresindependentes do comportamento de cuidado do indivíduo. A observação direta do comportamento, neste caso, é bastante indicada, por ser independente de indicadores fisiológicos. No entanto, a observação nem sempre é possível ou viável e o indivíduo pode modificar seu comportamento apenas por estar submetido ao contexto observacional.

3) Os itens dos instrumentos adquirem significados distintos para participante e pesquisador.

Já em 1957, Skinner destacava em seu livro Comportamento V erbal (em inglês, V erbal B ehavior) a importância do esclarecimento de significados, ou seja, a importância de estabelecer relação fidedigna entre 0 que se está dizendo e a topografia do comportamento relatado. De forma análoga, em pesquisassobre enfrentamento eadesão a tratamento, muitas vezes, a nomeação do comportamento pode ser equivocada, por parte do participante da pesquisa, ou ser ambígua, por parte do instrumento. Não é incomum que um indivíduo não compreenda as instruções descritas por seu médico, a respeito da medicação indicada, comprometendo a adesão ao tratamento e mesmo 0 autorrelato de comportamentos de cuidado (Arruda, 2002).

Por outro lado, a tradução e validação de inventários para caracterizar o enfrentamento pode se consolidar em escalas e questionários com frases incompreendidas pelo participante da pesquisa, resultando em um autorrelato falho a respeito das estratégias de enfrentamento adotadas (Seidl, Tróccoli \& Zannon, 2001).

\section{CON SIDERAÇÕES FINAIS}

Em seu livro Questões recentes na A nálise Comportamental, Skinner (1989/ 1991) ressalta que o comportamento de "fazer" algo sempre enfatiza as consequências - 0 efeito provocado sobre 0 mundo. O comportamento "fazer" pode ser efetivamente observado, mas, quando relatado, não há garantia de que exista correspondência fiel entre 0 comportamento real e o comportamento verbalizado. Da mesma forma, nas pesquisas em Psicologia da Saúde sobre adesão e enfrentamento, em que 0 autorrelato é majoritariamente utilizado sob a forma de inventários, questionários, escalas, diários e entrevistas, como garantir a correspondência entre 0 que é relatado pelo participante e o comportamento que realmente ocorre?

0 pesquisador tipicamente tem interesse em conhecer o comportamento do indivíduo e suas relações com o meio, mas não tem acesso direto a tal comportamento. Infelizmente, acorrespondência absoluta entre comportamento real ecomportamento relatado não pode ser garantida em todos os casos, mas 0 uso de metodologias combinadas pode diminuir de forma significativa os vieses do autorrelato. A aplicação de mais de um tipo de instrumento (entre escalas, entrevistas, questionários, inventários) confere mais fidedignidade ao autorrelato obtido, ao permitir que várias medidas sejam realizadas a respeito do comportamento em questão.

Além disso, a observação sistemática do comportamento é bastante indicada para o estudo da adesão e do enfrentamento, apesar de nem sempre ser possível estabelecer tal condição metodológica. Cabe ressaltar que utilizar a observação pode, por si só, modificar o comportamento do participante, fato que demanda mais cuidados metodológicos.

O preparo do pesquisador e o cuidado na aplicação de instrumentos padronizados eentrevistas são pontos cruciais para garantir que o participante compreenda não apenas as instruções do estudo e perguntas/ itens do instrumento, mas também para que seja fidedigno ao relatar seu próprio comportamento, evitando a falta de correspondência 
entre relato - comportamento e as respostas com viés do que seria socialmente desejável. São necessários cuidados específicos ao aplicar um instrumento padronizado ou realizar uma entrevista, tanto nas instruções quanto no feedback ao participante, para 0 pesquisador não emitir reforços generalizados que possam influenciar o relato do indivíduo.

$\mathrm{Na}$ pesquisa sobre adesão ao tratamento e enfrentamento, há um aspecto imensamente relevante a respeito do uso do autorrelato como possibilidade de tornar o indivíduo mais ativo em seu processo saúde-doença, que se constitui um dos objetivos indiretos da Psicologia da Saúde. Skinner (1989/1991) destaca, a respeito do autorrelato, que as pessoas são solicitadas a falar sobre o que estão fazendo ou por que o fazem e, ao responderem, podem tanto falar de si próprias quanto sobre outrem, provavelmente aumentando a auto-observação. Consequentemente, os comportamentos e autorregras também têm maior probabilidade de serem esclarecidos e, a partir daí, modificados, influenciando decisivamente a atuação do profissional em saúdejunto ao paciente.

Skinner (1957/ 1978) destaca, ainda, que o comportamento verbal autodescritivo é de interesse por muitas razões: somente através da aquisição de tal comportamento é que 0 falante pode tornar-se consciente do que está fazendo ou dizendo e por que o faz. D esta forma, é possível estabelecer as contingências que mantêm tal comportamento e atuar sobre elas.

Beckert (2002) afirma que a modelagem de descrições fidedignas do comportamento amplia comportamentos de auto-observação, discriminação e descrição do indivíduo, promovendo um autorrelato mais completo sobre comportamentos observados. A partir dos tatos e intraverbais que correspondam à descrição do mundo interno e externo do cliente, aumenta, assim, seu poder de observação sobre si mesmo e sobre o mundo (Ferster, 1979 apud Beckert, 2002).

Ao pesquisar temas como enfrentamento e adesão ao tratamento, torna-se crucial refletir também sobre a atuação do indivíduo como efetivamente ativo em seu processo saúde-doença, não como mero elemento passivo na situação. Essa efetivainserção em seu tratamento torna-se possível, entre outros aspectos contextuais e globais, a partir daauto-observação proporcionada pelo autorrelato. Entretanto, cuidados metodológicos precisam ser observados para garantir maior fidedignidade do relato sobre o próprio comportamento e melhor qualidade da pesquisa em Psicologia da Saúde.

Agradecimento: à Coordenação e A perfeiçoamento de Pessoal de Nível Superior CAPES, pelo apoio financeiro

\section{REFERÊNCIAS}

Antoniazzi, A. S., D ell'Aglio, D. D., \& Bandeira, D. R. (1998). O conceito de coping: Uma revisão teórica. Estudos de Psicologia, 3(2), 273-294.

Arruda, P. M. Exigências para adesão ao tratamento pediátrico de febre reumática $e$ diabetes Melitus Tipo 1 e estratégias de enfrentamento do cuidador. D issertação de Mestrado, Universidade de Brasília, Brasília.

Beckert, M. (2002). Correspondência: Q uando o objetivo terapêutico é o "digo o que faço e faço o que digo." In H. J. G uilhardi, M. B. B. P., Madi P. P., \& M. C. Q ueiroz (O rg.). Sobre comportamento e cognição: Aspectos teóricos, metodológicos e de formação em análise do comportamento e terapia cognitivista (10a ed., pp. 183-194). Santo André: ESETec.

Cerqueira, A. T. A. R. (2000). O conceito e metodologia de coping: existe consenso e necessidade? In R. R. Kerbauy (O rg.). Sobre comportamento e cognição: Aspectos teóricos, metodológicos e de formação em análise do comportamento e terapia cognitivista (5a ed., pp. 271-281). Santo André: ESETec.

Coyne, J. C., \& Racioppo, M. W. (2000). Never the twain shall meet? Closing the gap between coping research and clinical intervention research. American Psychologist, 55(6), 655-664.

De Rose, J. C. C. (2001). O relato verbal segundo a perspectiva da análise do comportamento: contribuições conceituais e experimentais. In R. A. Banaco (O rg.). Sobre comportamento e cognição: Aspectos teóricos, metodológicos e de formação em análise do comportamento e terapia cognitivista (pp. 140-153). Santo André: ESETec. 
Epstein, L. H., \& Cluss, P. A. (1982). A behavioral perspective on adherence to longterm medical regimens. Journal of Consulting and Clinical Psychology, 50(6), 950-971.

Ferster, C. B. (1979). A laboratory model of psychotherapy: The boundary between clinical practice and experimental psychology. In I. P. Sjöden, S. Bates \& W. S. D ockens (O rg.). Trends in behavior therapy (pp. 230-238). New York: Academic Press.

Folkman, S., Lazarus, R. S., G ruen, R. J., \& DeLonguis, A. (1986). Appraisal, coping, health status, and psychological symptoms. Journal of Personality and Social Psychology, 50(3), 571-579.

Lazarus, R., \& Launier, R. (1978). Stress related transactions between persons and environment. In Pervinla O., \& Lewis M. (Ed.). Perspectives in international psychology (pp. 287-232). New York: Plenum Press.

Lazarus, R. (1966). Psychological stress and the coping process. New York: McG rallHill.

Lazarus, R. S., \& Folkman, S. (1984). Stress, appraisal and coping. New York: Springer.

Malerbi, F. E. K. (2000). Adesão ao tratamento. In R. R. Kerbauy (O rg.). Sobre comportamento e cognição: Aspectos teóricos, metodológicos e de formação em análise do comportamento e terapia cognitivista (5a ed., pp. 144-151). Santo André: ESETec.

Matarazzo, J. D. (1980). Behavioral health and behavioral medicine: Frontiers for a new health psychology. American Psychologist, 35(9), 807-817.

Noyes, J. (1999). The impact of knowing your child is critically ill: A qualitative study of mothers' experiences. Journal of Advanced N ursing, 29(2), 427-435.

Seidl, E. M. F., Tróccoli, B. T., \& Zannon, C. M. L. C. (2001). Análise fatorial de uma medida de estratégias de enfrentamento. Psicologia: Teoria e Pesquisa, 17(3), 225-234.
Skinner, B. F. (1967). Ciência e comportamento humano. (J. C. Todorov \& R. Azzi Trans). Brasília: Editora da Universidade de Brasília. (O riginalmente publicado em 1953).

Skinner, B. F. (1978). 0 comportamento verbal. (M. P. Villalobos Trans). São Paulo: Cultrix. (O riginalmente publicado em 1957).

Skinner, B. F. (1991). Q uestões recentes na análise comportamental. (A. L. Néri Trans). São Paulo: Campinas. (O riginalmente publicado em 1989).

Recebido: 27/ 02/ 2009

Received: 02/ 27/ 2009

Aprovado: 30/ 04/ 2009

A pproved: 04/30/ 2009

Revisado: 31/ 07/ 2009

Reviewed: 07/ 31/ 2009 